\title{
Geometrical guidance and trapping transition of human sperm cells
}

\author{
A. Guidobaldi, ${ }^{1}$ Y. Jeyaram, ${ }^{2}$ I. Berdakin, ${ }^{3}$ V. V. Moshchalkov, ${ }^{2}$ C. A. Condat, ${ }^{3}$ V. I. Marconi, ${ }^{3}$ L. Giojalas, ${ }^{1}$ and A. V. Silhanek ${ }^{4}$ \\ ${ }^{1}$ Instituto de Investigaciones Biológicas y Tecnológicas, CONICET and Centro de Biología Celular y Molecular, Universidad Nacional de \\ Córdoba, Ciudad Universitaria, 5000-Córdoba, Argentina \\ ${ }^{2}$ Institute for Nanoscale Physics and Chemistry, KU Leuven, B-3001 Leuven, Belgium \\ ${ }^{3}$ Facultad de Matemática, Astronomía y Física, Universidad Nacional de Córdoba and IFEG-CONICET, X5000HUA Córdoba, Argentina \\ ${ }^{4}$ Département de Physique, Université de Liège, B-4000 Sart Tilman, Belgium
}

(Received 19 December 2013; revised manuscript received 6 February 2014; published 28 March 2014)

\begin{abstract}
The guidance of human sperm cells under confinement in quasi-2D microchambers is investigated using a purely physical method to control their distribution. Transport property measurements and simulations are performed with diluted sperm populations, for which effects of geometrical guidance and concentration are studied in detail. In particular, a trapping transition at convex angular wall features is identified and analyzed. We also show that highly efficient microratchets can be fabricated by using curved asymmetric obstacles to take advantage of the spermatozoa specific swimming strategy.
\end{abstract}

DOI: 10.1103/PhysRevE.89.032720 PACS number(s): 87.17.Jj, 05.40.-a, 87.17.Aa, 87.18.Hf

\section{INTRODUCTION}

Understanding sperm dynamics under confining microgeometries is of primary importance for future biomedical applications and represents a major challenge both from the basic biophysics and the complex fluids points of view. Indeed, confinement effects studied by in vitro assays with microfluidic chambers may help us to understand the dynamics of sperm in the narrow oviduct passage traversed on their journey to the oocyte, or to develop biomedical devices for improving the treatment of infertile couples. Unfortunately, our knowledge of the swimming cell motilities in unbounded media cannot be directly extrapolated to their behavior in complex and space-limited environments, due to the close proximity to the surfaces and the consequent boundary effects. In the case where the constrictions are of similar size as the sperm, i.e., a few tens of micrometers, confined cells may undergo substantial changes in their locomotion habits, as already reported for other self-propelled microorganisms [1-3]. It has been shown that microswimmers with very different propulsion systems are similarly attracted to the walls and tend to swim parallel to the surface [2,4-11]. It is believed that this attractive force has hydrodynamic origin although other possible mechanisms have been proposed [12-14]. Several models have been introduced to describe the swimming along infinite planar surfaces [8,9,12,15-19]. Interestingly, the observed cell-wall attraction, as shown in Fig. 1, has motivated the design of ratchet devices that guide and sort self-propelled cells using asymmetric obstacles [20,21] as well as microfluidic devices aiming to either increase sperm cell quality or enhance their concentration [22-24]. The creation of inhomogeneous distributions of swimmer populations via asymmetric obstacles has been shown to be particularly efficient for run-and-tumble bacteria [20,25-28] and numerous theoretical treatments are available to account for the effects of asymmetric obstacles on active particles distributions [29-39]. Although larger and generally faster than bacteria, a similar rectification effect should, in principle, be observed for human sperm cells. However, a distinct property of sperm cells is their specific swimming strategy. Since spermatozoa do not tumble, their detachment from the wall, in diluted samples, will presumably proceed from effects other than tumbling, such as the observed flagellar beating of bull spermatozoa near corners [21], rotational diffusion [8], or sporadic cell-cell collisions [40].

In this work, we show experimentally and numerically that asymmetric designs as those previously used to generate inhomogeneous bacterial distributions need to be revisited when dealing with sperm cells. Indeed, V-shaped asymmetric pillar arrays introduced to rectify bacterial displacement [20] lead to sperm trapping near the obstacle apices. A high concentration of sperm cells is then obtained at every convex angle at the expense of emptying the space away from the confining walls. This undesired effect renders the chambers with V-shaped obstacles of limited use in biomedical applications. In order to avoid this trapping mechanism leading to extreme cell aggregation, we introduce rounded and asymmetric $\cup$-shaped funnel arrays and rounded box corners. We demonstrate that, by building a row of $U$-shaped funnels facing a row of $\cap$-shaped funnels, it is possible to obtain a high concentration of uniformly distributed sperm cells in the region confined between the rows. Numerical simulations based on a minimal model, which reproduces qualitatively the experimental findings, allows us to capture and identify the essential physics and predict the influence of several geometrical parameters on the design. This work is then the result of a strong positive feedback among soft-lithography microfabrication, biological measurements, and phenomenological modeling without adjustable parameters.

\section{FABRICATION OF HYDROSTATIC MICROFLUIDIC CHAMBERS}

The quasi-2D nanoliter chambers were prepared in SU-8 or EPOCLAD negative optical resists deposited on glass substrates. The materials were chosen to ensure (a) optical transparency allowing transmission microscopy imaging, (b) high aspect ratio structures (height/width $>20$ ), (c) biocompatibility, and (d) watertightness. Both epoxies show no particular differences concerning biocompatibility within the time frame of the experiments, although SU-8 provided higher resolution for nanofabrication. We have observed no major 


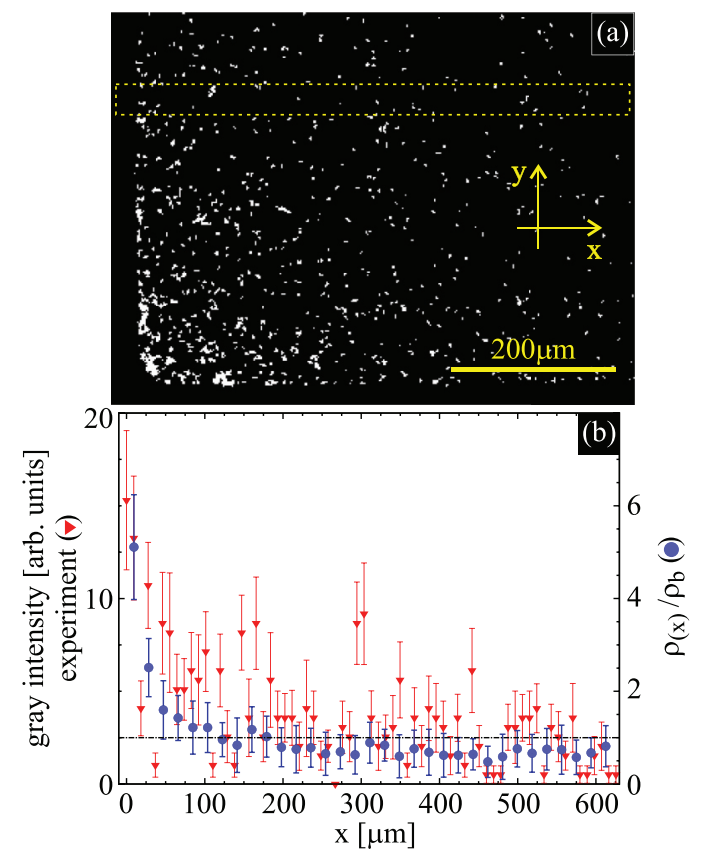

FIG. 1. (Color online) (a) Microscopy image of a twodimensional sperm distribution close to the chamber corner. Depth $L_{z}=20 \mu \mathrm{m}$. The walls of the container become apparent by the accumulation of sperm cells. (b) Quantification of the sperm distribution proportional to the gray intensity. Experimental data is indicated with triangles and numerical results with circles. The dashed line corresponds to the average concentration. For more information see the figure in the Supplemental Material [41].

differences between both epoxies concerning the physical response of the sperm cells, thus evidencing the robustness of the main results of this work.

\section{SPERM PREPARATION AND MANIPULATION}

Spermatozoa were separated from the seminal plasma by a discontinuous Percoll gradient [42]. The highly motile sperm population was adjusted to $10^{7}$ cells $/ \mathrm{ml}$ with HAMF10 medium containing $25 \mathrm{mM}$ Hepes and L-glutamine, supplemented with $1 \%$ human albumin. Then, the sperm were loaded with $1 \mu \mathrm{M}$ of Fluo4-AM for at least $15 \mathrm{~min}$ and then kept in an incubator at $37^{\circ} \mathrm{C}$ with $5 \% \mathrm{CO}_{2}$ in air until their use. The chambers were loaded with $500 \mathrm{nl}$ of sperm suspension over the well and covered by sliding a handmade $3 \times 3-\mathrm{mm}^{2}$ coverslip to avoid air bubble formation. To achieve a seal, excess liquid was absorbed with paper. The edges were covered with mineral oil to prevent air entry. The sperm movement was recorded by fluorescent videomicroscopy using a digital camera connected to an inverted microscope (Nikon, USA). Recordings of tracks were performed at $25 \mathrm{~Hz}$ with the BR Nis Elements software and the image analysis was made with ImageJ free software.

\section{MODEL}

The dynamics of the sperm cell population is represented by the overdamped motion of $N$ self-propelled discs moving in two dimensions and determined by the following equations
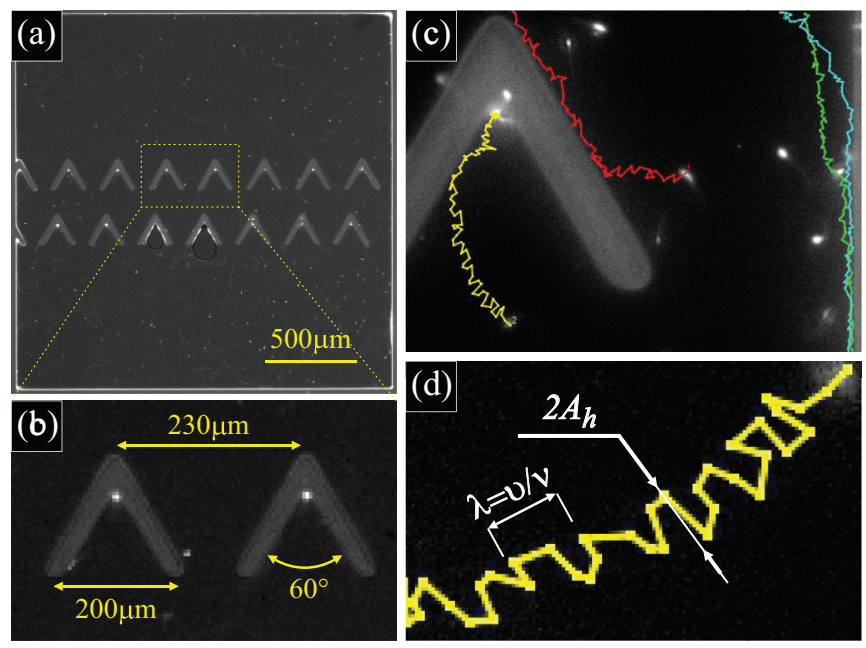

FIG. 2. (Color online) Human sperm trapping near the apices of a V-shaped ratchet. (a) Chamber with a double row of V-shaped obstacles similar to those used for bacterial motion rectification, clearly showing accumulation at all apices. (b) Geometrical parameters and detail of the sperm accumulation (brighter dots) at the $\mathrm{V}$-apices. (c) High magnification details of the sperm cells behavior: cell trapping at a convex angle and swimming along walls. Some tracked cell trajectories are shown with colored lines. See movie in Ref. [41]. (d) Cell head oscillation: amplitude $A_{h}$ and wavelength $\lambda=v / v$.

of motion,

$$
\begin{aligned}
& \dot{\boldsymbol{r}}_{i}=v_{i} \hat{e}_{i}^{\|}+A_{h} \omega_{i} \cos \left(\omega_{i} t\right) \hat{e}_{i}^{\perp} \\
& \dot{\varphi}_{i}=\sqrt{2 D_{r}^{i}} \chi,
\end{aligned}
$$

where the vector $\boldsymbol{r}_{i}$ represents the position of the center of the $i$ th disk, $\varphi_{i}$ is its swimming direction measured from the $x$ axis, and $\chi$ is a zero mean gaussian white noise with unit variance. The unit vectors $\hat{e}_{i}^{\|}=\cos \left(\varphi_{i}\right) \hat{e}_{x}+\sin \left(\varphi_{i}\right) \hat{e}_{y}$ and $\hat{e}_{i}^{\perp}=-\sin \left(\varphi_{i}\right) \hat{e}_{x}+\cos \left(\varphi_{i}\right) \hat{e}_{y}$ are parallel and perpendicular to the swimming direction, respectively. The amplitude $A_{h}$ and frequency of oscillation $\omega_{i}=2 \pi v_{i}$ mimic the oscillations of the sperm head as shown in Fig. 2(d). The parameters characterizing the motility of the cells, i.e., the velocity $v_{i}$, $\omega_{i}$, and rotational diffusion coefficient $D_{r}^{i}$, follow the gaussian distribution that best fits the measured distribution obtained by analyzing the longest 94 measured tracks. The mean value and the standard deviation for these distributions are $\langle v\rangle=$ $28 \mu \mathrm{m} / \mathrm{s}, \sigma_{v}=4 \mu \mathrm{m} / \mathrm{s},\langle v\rangle=10 \mathrm{~Hz}, \sigma_{v}=1 \mathrm{~Hz}$, and $\left\langle D_{r}\right\rangle=$ $0.01 \mathrm{rad}^{2} / \mathrm{s}, \sigma_{D_{r}}=0.01 \mathrm{rad}^{2} / \mathrm{s}$. For all swimmers, we consider the same, experimentally obtained, oscillation amplitude $\left\langle A_{h}\right\rangle=1.5 \mu \mathrm{m}$. In addition, we have also considered a normal distribution of head sizes with $\left\langle R_{h}\right\rangle=2.5 \mu \mathrm{m}$ and $\sigma_{R_{h}}=0.1$ $\mu \mathrm{m}$. We consider only steric interactions among swimmers (see sketch and panel (a) in the Supplemental Material [41]), as suggested by the experimental observation that cell-cell collisions are very rare events in our diluted system. A repulsive force acts on each swimmer upon contact. This force pulls the swimmers apart preventing cell-cell overlaps but the swimmers recover their original swimming directions after collisions (our return to the precollision swimming headings 
is similar to the results observed in Ref. [43]). Similarly, a repulsive force also acts on the cells when they collide with the walls (see sketch and panel (b) in the Supplemental Material [41]). However, in this case, cells do change their direction of motion in order to swim parallel to the wall. The inclusion of the head oscillation in the model becomes relevant at this point to phenomenologically account for the long residence time of the cells near the walls. During the half period of the oscillation when the head moves away from the wall, the cell may change its direction due to rotational diffusion and then escape, otherwise the head hits the wall again and the cycle restarts from the beginning (see panel (b) in Ref. [41]). The probability of leaving the wall results then proportional to $D_{r}$ and $A_{h}$. The stochastic equations of motion were integrated with a first-order method. All the simulation results were averaged over 50-100 initial conditions. Cells trajectories, surface accumulation, corner trapping, average densities in the lower chamber (easy direction of motion), $\rho_{e}$, average densities in the upper chamber (hard direction), $\rho_{h}$, and rectification effects were calculated, with the rectification defined as $r=\rho_{e} /\left(\rho_{e}+\rho_{h}\right)$.

\section{SPERM ACCUMULATION NEAR WALLS}

Spermatozoa were loaded into a well of area $2 \times 2 \mathrm{~mm}^{2}$ and depth $20 \mu \mathrm{m}$, allowing them to swim in an effectively two-dimensional region. In Fig. 1(a) we show a snapshot of one chamber corner in the $x y$ plane. We observe that sperm cells tend to accumulate near the boundaries while maintaining a fairly homogeneous distribution far from them. In order to quantify more precisely the spatial dependence of the cell distribution, we have considered five nonoverlapping rectangular boxes such as the one delimited by a dashed line in Fig. 1(a). These boxes, with randomly chosen locations in the region between 250 and $600 \mu \mathrm{m}$ above the corner [44] shown in the figure, were divided into vertical bins of 10 pixels width $(\sim 18 \mu \mathrm{m})$. We then averaged the intensity over each bin and plotted the result in Fig. 1(b). Clearly, a monotonic increment of the cell density is observed as we approach the wall, reaching a density of about 5 times that in the middle of the box. We remark that the cell accumulation near the walls was measured in the 2D $x y$ plane and may not necessarily follow the spatial dependence reported using sperm of other mammals on a drop of fluid compressed between two glass slides [8]. On the contrary, in our case the wall height, $L_{z}$ is just $\sim 20 \mu \mathrm{m}$, i.e., about twice the largest dimension of the sperm head. In Fig. 1(b) we also plot the numerical results obtained using experimental values characterizing cell motility (see Fig. 2). Note the good qualitative agreement, given that there are no free parameters. Due to the finite size of the system, the accumulation near the walls generates cell depletion away from them. The cell density decays approximately as the inverse square root of the distance to the wall.

\section{SPERM TRAPPING IN V-SHAPED WALLS}

The observation that walls attract sperm cells so that they swim close to them encouraged us to design cell sorters following the layouts already tested for bacteria [20]. One of these designs consisting of a chamber divided by

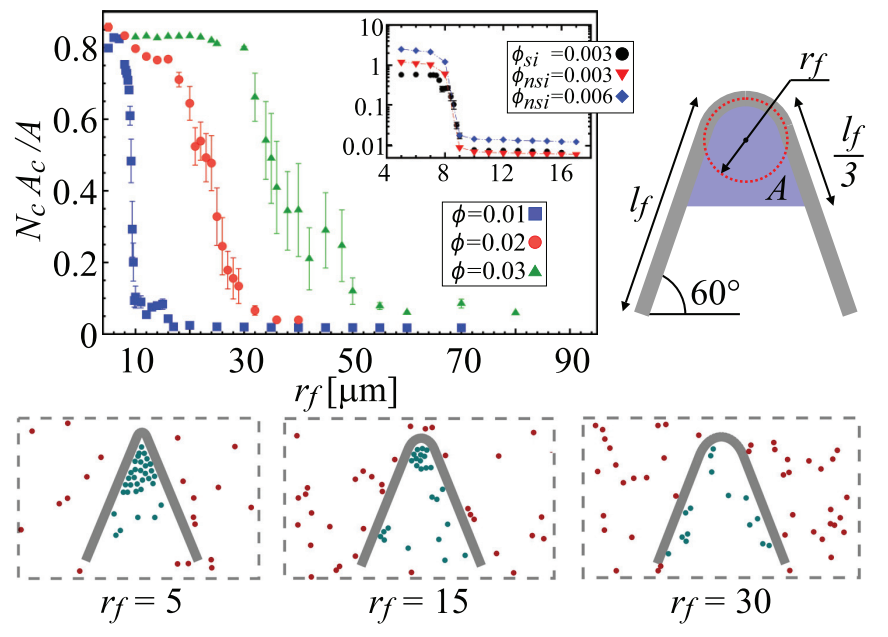

FIG. 3. (Color online) Numerical results of the obstacle curvature effects. Main panel: fraction of the shaded area $A$ in the scheme on the right occupied by sperm cells vs. the radius of curvature, for the indicated sperm densities $\phi$ and $l_{f}=100 \mu \mathrm{m}$. Inset: results at lower $\phi$, with steric interactions (si) and with no steric interactions (nsi). Lower panels: Snapshots for $\phi=0.02$ and different $r_{f}$ values. $N_{c}$ is the average number of cells found in $A$. A density of $\phi=0.01$ corresponds to a cell concentration of $\rho_{b}=6 \times 10^{6}$ cells $/ \mathrm{ml}$.

two rows of asymmetric V-shaped obstacles is shown in Fig. 2(a). When this chamber is homogeneously inoculated with sperm cells, the cells are guided by the walls, and the asymmetric shape of the funnels gives rise to a higher population density in the lower chamber. Unfortunately, this design leads to cell directioning but not as efficient as expected due to unwanted cell concentration near the convex angles of the V-shaped obstacles, Figs. 2(a)-2(c). We simulated this phenomenon, keeping the apex angle fixed while changing the rounding (radius of curvature) of the $\mathrm{V}$ tip in order to look for a trapping-detrapping transition. The accumulation of sperm cells observed near the V-shaped corners and all other corners in the chamber is a direct consequence of their specific swimming strategy, a persistent progressive forward movement (see the movie in the Supplemental Material [41]). Our simulations were performed under periodic boundary conditions for a single V-shaped obstacle as illustrated by the scheme in Fig. 3 using various values for the tip curvature. The average sperm surface density $\phi$ is calculated as $\phi=$ $N A_{c} /\left(L_{x} L_{y}\right)$, where $A_{c}$ is the individual cell area and $L_{x} L_{y}$ the total simulated area with $L_{x}=L_{y}=1000 \mu \mathrm{m}$. The main panel of Fig. 3 shows the average occupied area in $A$ as a function of the radius of curvature $r_{f}$, for three values of $\phi$ and taking $l_{f}=100 \mu \mathrm{m}$. Note in the inset of Fig. 3 the sudden detrapping transition obtained at $r_{f} \sim 8 \mu \mathrm{m}$ for a $\phi$ value one order of magnitude smaller, $\phi=0.003$. The same result is obtained if we turn off the cell-cell interaction (no steric interactions, nsi). In this way, we force the system to remain in the single-cell regime independently of the density of cells, and we expect that the trapping transition would take place at $8 \mu \mathrm{m}$, as indeed is confirmed by the two densities showed in the inset. This critical value of the curvature of $8 \mu \mathrm{m}$ is not surprising since an individual cell needs a space of the order of $2 R_{h}+2 A_{h} \sim 8 \mu \mathrm{m}$ to maneuver out of the corner. 
The transition in Fig. 3 exhibits other two properties. First, it moves to higher values of $r_{f}$ as we increase the density: since the cells are on the average pushing toward the vertex, increasing their number in the apex region leads to an increase in the jamming probability, hindering detachment. Second, it is sharp: If we increment $r_{f}$ for a given value of $\phi$, there is a $r_{f}$ value for which one of the cells has room enough to detach from the jam. Detachment of the first cell leaves more room for the rest, facilitating further corner evacuation. Simulations performed for different $l_{f}$ show that by increasing $l_{f}$ the trapping transition shifts to larger values of $r_{f}$. Based on the fact that the legs of the V-shaped obstacle act as collectors and guides for the swimmers, it is expected that the trapping becomes stronger when the legs are elongated. Indeed, the rate of disks coming into the obstacle increases with $l_{f}$, whereas the escaping rate is, to first order, independent of $l_{f}$.

\section{SPERM DETRAPPING AND RECTIFICATION IN U-SHAPED WALLS}

It is clear from both experiment and simulation that rounding off the tips of the $\mathrm{V}$-shaped obstacles will permit us to reorient the cells toward the bulk of the reservoirs in order to maintain a more homogeneous distribution. We thus designed a chamber where a single row of V-shaped obstacles was replaced by a line of $\cup$-shaped pillars. To optimize the design, we first simulated the cell dynamics in a chamber containing a row of round-shaped funnels [see inset of Fig. 4(a)] with eight gaps of size $l_{g}=10 \mu \mathrm{m}$ and wall width $w=25 \mu \mathrm{m}$ for several curvature radii $r_{f}$, adapting $L_{x}$ and leaving $L_{y}$ fixed at $1885 \mu \mathrm{m}$. Figure 4(a) exhibits the numerical results, with the rectification $r$ being the normalized steady-state cell number

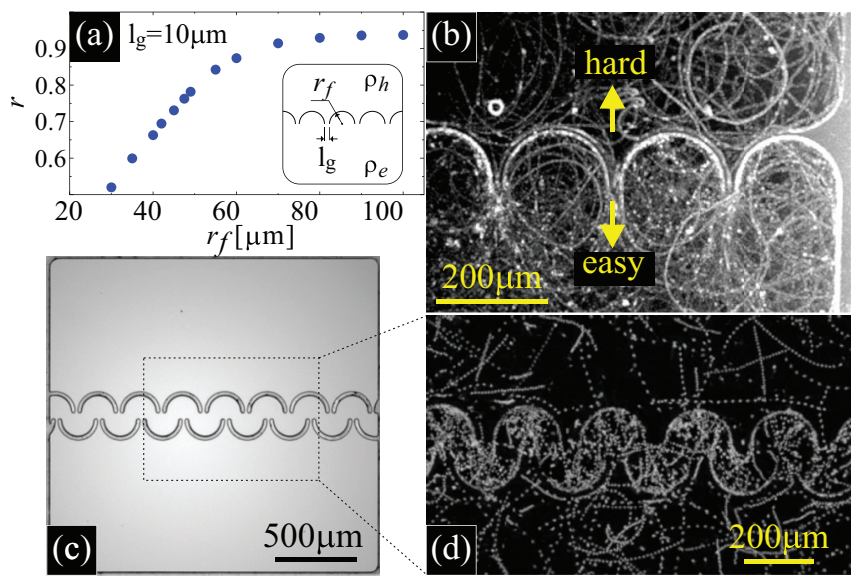

FIG. 4. (Color online) (a) Simulated sperm rectification in an 8-gap chamber as a function of the radius of curvature. The rectification is close to $90 \%$ for curvature radii longer than $80 \mu \mathrm{m}$. Inset: sketch of the simulated ratchet system with rounded obstacles. (b) A single row of $\cap$-shaped funnels guides sperm cells in the easy direction of motion, i.e., toward the lower half of the chamber. (c) A combination of $\cup$-shaped and $\cap$-shaped funnels was fabricated. (d) The microarchitecture in (c) satisfies our initial goal: a limited region of highly concentrated sperm cells without corner accumulation. Numerical and experimental density, $\rho_{b}=10^{7}$ cells $/ \mathrm{ml}$. Microscopy images in (b) and (d) represent time projections evidencing the sperm trajectories. in the lower (easy) chamber. The rectification increases in a roughly linear fashion until it saturates at $r_{f} \sim 80 \mu \mathrm{m}$. The constant cell leakage through the gaps prevents $100 \%$ rectification efficiency and instead a $90 \%$ rectification efficiency is observed. Following this template, we fabricated microchambers with a row of round-shaped funnels, as shown in Fig. 4(b), with either four or eight gaps, $r_{f} \approx 100 \mu \mathrm{m}$ and $l_{g} \approx 15 \mu \mathrm{m}$. In Fig. 4(b) we track individual cell trajectories by projecting the maximum intensity of 100 frames $(4 \mathrm{~s})$. From this image we see that sperm cells often follow circular paths as pointed out in previous reports on quasi-2D confined systems $[6,7,10]$.

The collected trajectories evidence the existence of an enhanced cell density in the lower chamber, a consequence of the broken funnel symmetry. Furthermore, by using a row of $\cup$-shaped funnels facing a row of $\cap$-shaped funnels as shown in Figs. 4(c) and 4(d), we achieve high sperm concentrations in the space between the rows without corner trapping. These high cell concentrations could be useful for creating a sperm jet or harvesting sperm cells from a reservoir into a narrow channel. The details of the chambers fabrication, such as sharp edges and the sameness in the obstacles, can be appreciated in Fig. 4(c).

We quantified the rectification efficiency by following two different experimental approaches. First we counted the number of cells crossing each gap in Fig. 4(b) in the easy (e) and hard (h) directions within a timeframe of two minutes.

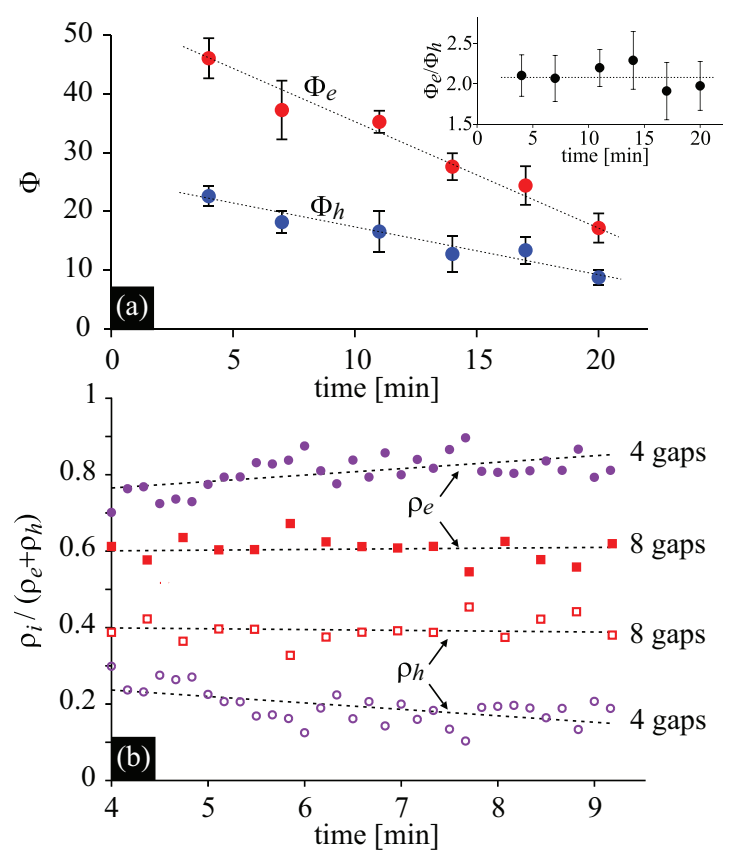

FIG. 5. (Color online) Quantifying human sperm rectification. (a) Sperm cell fluxes in the easy $\left(\Phi_{e}\right)$ and hard $\left(\Phi_{h}\right)$ ratchet directions vs. time in the experiment shown in Fig. 4(b). $\Phi$ is calculated measuring the number $N_{c}$ of crossing cells during two minutes. Flux decay is due to cell death (see text). Inset: relative sperm fluxes. (b) Steady-state relative concentrations on both sides of the obstacle wall for geometries with 4 and 8 gaps (circles and squares, respectively). The $y$-axis label $\rho_{i}$ must be read as $\rho_{e}$ or $\rho_{h}$, as corresponds to the curves labeled as $\rho_{i}$ inside the panel. Time is measured starting 4 min after the chamber is closed. Early times are omitted because of experimental constraints. 
After averaging over five contiguous gaps, the resulting fluxes, $\Phi_{e}$ and $\Phi_{h}$, are shown in Fig. 5(a) as functions of time. We observe that $\Phi_{e}$ systematically exceeds $\Phi_{h}$, thus showing that the asymmetric structure operates as a geometrical ratchet or rectifier, suggesting a purely physical method for controlling sperm cell dynamics and distribution. The linear decay of $\Phi_{e}$ and $\Phi_{h}$ may reflect the death rate of cells in a somewhat hostile 2D confining habitat after $15 \mathrm{~min}$. Irrespective of cell death, we show in the inset of Fig. 5(a) that the ratio of the swimmer fluxes remains practically constant, being twice as likely that a particle moves in the easy direction as in the hard direction. The second approach consists of directly measuring the density of sperm cells in a well-delimited region covering the same area on each side of the chamber. The result of this study is summarized in Fig. 5(b). Interestingly, while a moderate cell separation is observed for an eight-gap chamber, the sorting efficiency is substantially increased if a narrower chamber with only four gaps is used, in agreement with the trend of numerical simulations. We believe this to be due to the larger perimeter-to-area ratio of the smaller chamber, which favors easier wall capture.

\section{CONCLUSION}

In summary, we performed original microratchet experiments and numerical studies on human sperm cells. Our simple and minimal phenomenological model captures the essential observed sperm behavior and gives excellent predictions without adjustable parameters. We started by verifying that human sperm cells are attracted to boundaries, obtaining the concentration variation near a wall with a very good agreement between experiment and simulation. We found experimentally that sperm cells are trapped near the apices of angular wall features, thus rendering V-shaped structures less suitable for uniform sperm cell directioning and separation. We then studied numerically the trapping-detrapping transition that occurs when we change the curvature radius of an angular feature, finding that this transition is remarkably sharp. Using our predicted geometries, which, incidentally, were in general agreement with very recent theoretical works [45-47], rows of U-shaped obstacles were fabricated and a high sperm cell concentration was observed, demonstrating that the interaction of the cells with the rounded walls leads to the rectification and control of the sperm movement without trapping the cells. Our noninvasive procedure is an alternative efficient method to achieve sperm sorting [48,49] and directioning [40] in channels. We have also shown that a characterization of the specific sperm cells strategy for swimming along walls, its behavior near corners and along edges, together with the knowledge of the motility parameters under 2D confinement, will be crucial for designing and modeling efficient biomedical devices. In addition, we hope that our findings will shed new light on the understanding of the puzzling microswimmer-wall interactions $[8,9,11,12,15-19,21,40]$.

\section{ACKNOWLEDGMENTS}

We acknowledge financial support from CONICET, MINCyT, SeCyT-UNC (Argentina), and the FNRS-CONICET bilateral project $(\mathrm{V} 4 / 325 \mathrm{C})$. This work was also partially supported by the FNRS, the Methusalem Funding, and the FWO-Vlaanderen (Belgium). Authors are grateful to R. Soto for the critical reading of the manuscript and E. Altshuler for useful discussions.
[1] G. Miño, T. E. Mallouk, T. Darnige, M. Hoyos, J. Dauchet, J. Dunstan, R. Soto, Y. Wang, A. Rousselet, and E. Clement, Phys. Rev. Lett. 106, 048102 (2011).

[2] P. Frymier, R. M. Ford, H. C. Berg, and P. Cummings, Proc. Natl. Acad. Sci. USA 92, 14861 (1995).

[3] J. Männik, R. Driessen, P. Galajda, J. E. Keymer, and C. Dekker, Proc. Natl. Acad. Sci. USA 106, 14861 (2009).

[4] L. Rothschild, Nature 198, 1221 (1963).

[5] H. Winet, G. S. Bernstein, and J. Head, J. Reprod. Fertil. 70, 511 (1984).

[6] J. Cosson, P. Huitorel, and C. Gagnon, Cell Motil. Cytoskeleton 54, 56 (2003).

[7] D. M. Woolley, Reproduction 126, 259 (2003).

[8] G. Li and J. X. Tang, Phys. Rev. Lett. 103, 078101 (2009).

[9] A. P. Berke, L. Turner, H. C. Berg, and E. Lauga, Phys. Rev. Lett. 101, 038102 (2008).

[10] M.d.C. Lopez-Garcia, R. L. Monson, K. Haubert, M. B. Wheeler, and D. J. Beebe, Biomed. Microdevices 10, 709 (2008).

[11] K. Drescher, J. Dunkel, L. H. Cisneros, S. Ganguly, and R. E. Goldstein, Proc. Natl. Acad. Sci. USA 108, 10940 (2011).

[12] D. J. Smith, E. A. Gaffney, H. Shum, H. Gadêlha, and J. Kirkman-Brown, Biophys. J. 100, 2318 (2011).

[13] E. A. Gaffney, H. Gadêlha, D. J. Smith, J. R. Blake, and J. C. Kirkman-Brown, Annu. Rev. Fluid Mech. 43, 501 (2011).
[14] J. Elgeti, U. B. Kaupp, and G. Gompper, Biophys. J. 100, 2321 (2011).

[15] J. Elgeti and G. Gompper, Europhys. Lett. 85, 38002 (2009).

[16] J. Elgeti, U. B. Kaupp, and G. Gompper, Biophys. J. 99, 1018 (2010).

[17] J. Elgeti and G. Gompper, Europhys. Lett. 101, 48003 (2013).

[18] D. J. Smith, E. A. Gaffney, J. R. Blake, and J. Kirkman-Brown, J. Fluid Mech. 621, 289 (2009).

[19] J. Dunstan, G. Miño, E. Clement, and R. Soto, Phys. Fluids 24, 011901 (2012).

[20] P. Galajda, J. Keymer, P. Chaikin, and R. Austin, J. Bacteriol. 189, 8704 (2007).

[21] V. Kantsler, J. Dunkel, M. Polin, and R. E. Goldstein, Proc. Natl. Acad. Sci. USA 110, 1187 (2013).

[22] Y. A. Chen, Z. W. Huang, F. S. Tsai, C. Y. Chen, C. M. Liu, and A. M. Wo, Microfluid. Nanofluid. 10, 59 (2011).

[23] S. Tasoglu, H. Safaee, X. Zhang, J. L. Kingsley, P. N. Catalano, U. A. Gurkan, A. Nureddin, E. Kayaalp, R. M. Anchan, R. L. Maas, E. Tuzel, and U. Demirci, Small 9, 3374 (2013).

[24] K. Matsuura, T. Uozumi, T. Furuichi, I. Sugimoto, M. Kodama, and H. Funahashi, Fertil. Steril. 99, 400 (2013).

[25] S. E. Hulme, W. R. DiLuzio, S. S. Shevkoplyas, L. Turner, M. Mayer, H. C. Berg, and G. M. Whitesides, Lab Chip 8, 1888 (2008). 
[26] L. Angelani, R. Di Leonardo, and G. Ruocco, Phys. Rev. Lett. 102, 048104 (2009).

[27] S. Y. Kim, E. S. Lee, H. J. Lee, S. Y. Lee, S. K. Lee, and T. Kim, J. Micromech. Microeng. 20, 095006 (2010).

[28] E. Altshuler, G. Miño, C. Pérez-Penichet, L. del Río, A. Lindner, A. Rousselet, and E. Clément, Soft Matter 9, 1864 (2013).

[29] M. B. Wan, C. J. Olson Reichhardt, Z. Nussinov, and C. Reichhardt, Phys. Rev. Lett. 101, 018102 (2008).

[30] J. Tailleur and M. E. Cates, Eur. Phys. Lett. 86, 60002 (2009).

[31] I. Berdakin, Y. Jeyaram, V. V. Moshchalkov, L. Venken, S. Dierckx, S. J. Vanderleyden, A. V. Silhanek, C. A. Condat, and V. I. Marconi, Phys. Rev. E 87, 052702 (2013).

[32] I. Berdakin, A. V. Silhanek, H. N. Moyano Cortéz, V. I. Marconi, and C. A. Condat, Central Eur. J. Phys. 11, 1653 (2013).

[33] P. K. Ghosh, V. R. Misko, F. Marchesoni, and F. Nori, Phys. Rev. Lett. 110, 268301 (2013).

[34] C. Reichhardt and C. J. Olson Reichhardt, Phys. Rev. E 88, 062310 (2013).

[35] B. Q. Ai, Q. Y. Chen, Y. F. He, F. G. Li, and W. R. Zhong, Phys. Rev. E 88, 062129 (2013).

[36] S. Martens, G. Schmid, A. V. Straube, L. Schimansky-Geier, and P. Hänggi, Eur. Phys. J.: Special Topics 222, 2453 (2013).

[37] G. Suárez, M. Hoyuelos, and H. O. Mártin, Phys. Rev. E 88, 052136 (2013).

[38] X. Zheng, B. tenHagen, A. Kaiser, M. Wu, H. Cui, Z. Silber-Li, and H. Lowen, Phys. Rev. E 88, 032304 (2013).

[39] M. C. Marchetti, J. F. Joanny, S. Ramaswamy, T. B. Liverpool, J. Prost, M. Rao, and R. A. Simha, Rev. Mod. Phys. 85, 1143 (2013).
[40] P. Denissenko, V. Kantsler, D. J. Smith, and J. Kirkman-Brown, Proc. Natl. Acad. Sci. USA 109, 8007 (2012).

[41] See Supplemental Material at http://link.aps.org/supplemental/ 10.1103/PhysRevE.89.032720 for sperm accumulation and motion along walls as well as corner accumulation (figure and movie) and for a sketch of the collisions.

[42] R. J. Aitken and J. S. Clarkson, J. Androl. 9, 367 (1988).

[43] T. Ishikawa, G. Sekiya, Y. Imai, and T. Yamaguchi, Biophys. J. 93, 2217 (2007).

[44] In order to avoid the reinforced boundary effects at the corner of the chamber, the spatial distributions of sperm cells were done starting $250 \mu \mathrm{m}$ away from the corner. The use of five different distributions calculated at random positions allows us to estimate the error bar of each point. It is important to note that a $1 \mathrm{D}$ reduced distribution along the $x$ axis or $y$ axis, give very similar spatial dependencies, showing the robustness of the results.

[45] A. Kaiser, H. H. Wensink, and H. Löwen, Phys. Rev. Lett. 108, 268307 (2012).

[46] J. Deseigne, S. Leonard, O. Dauchot, and H. Châte, Soft Matter 8, 5629 (2012).

[47] A. Kaiser, K. Popowa, H. H. Wensink, and H. Löwen, Phys. Rev. E. 88, 022311 (2013).

[48] B. S. Cho, T. G. Schuster, X. Zhu, D. Chang, G. D. Smith, and S. Takayama, Anal. Chem. 75, 1671 (2003).

[49] D. B. Seo, Y. Agca, Z. C. Feng, and J. K. Critser, Microfluid. Nanofluid. 3, 561 (2007). 